\title{
Low-Power Direct Resistive Sensor-to-Microcontroller Interfaces
}

\author{
Oscar López-Lapeña, Ernesto Serrano-Finetti, and Oscar Casas, Member, IEEE
}

\begin{abstract}
This paper analyzes the energy consumption of direct interface circuits where the data conversion of a resistive sensor is performed by a direct connection to a set of digital ports of a microcontroller $(\mu \mathrm{C})$. The causes of energy consumption as well as their relation to the measurement specifications in terms of uncertainty are analyzed. This analysis yields a tradeoff between energy consumption and measurement uncertainty, which sets a design procedure focused on achieving the lowest energy consumption for a given uncertainty and a measuring range. Together with this analysis, a novel experimental setup is proposed that allows one to measure the $\mu \mathrm{C}$ 's timer quantization uncertainty. An application example is shown where the design procedure is applied. The experimental results fairly fit the theoretical analysis, yielding only $5 \mu \mathrm{J}$ to achieve nine effective number of bits (ENOB) in a measuring range from 1 to $1.38 \mathrm{k} \Omega$. With the same ENOB, the energy is reduced to $1.9 \mu \mathrm{J}$ when the measurement limits are changed to 100 and $138 \mathrm{k} \Omega$.
\end{abstract}

Index Terms-Energy consumption, microcontrollers $(\mu \mathrm{C})$, powerless sensor nodes, resistive sensors, radio-frequency identifier, sensor electronic interface.

\section{INTRODUCTION}

$\mathbf{T}$ HE reduction of power consumption is an important requirement in the design of autonomous sensors to improve their autonomy or to reduce the cost of power supply energy harvester systems. It is one of the main design bottlenecks for incoming new technologies such as powerless sensor nodes integrated in passive radio-frequency identifier systems [1], [2]. Although the main research efforts have focused on lowering the energy spent by the communication system [3], the measurement process can spend more energy whenever not all the measurement data are transmitted.

The analysis of the energy consumption of a sensor interface depends on the sensor nature. In the case of resistive sensors, conventional interfaces use either a voltage divider or a Wheatstone bridge together with an amplifier, a low-pass filter, and an analog-to-digital converter (ADC) as those that are currently embedded in a microcontroller $(\mu \mathrm{C})$. The power consumption of such circuits was previously analyzed in [4]. Nevertheless, some low-cost $\mu \mathrm{Cs}$ do not provide an ADC or its effective number of bits (ENOB) is not good enough for a specific application. In those cases, it is possible to use an

Manuscript received March 9, 2015; revised June 19, 2015; accepted August 14, 2015. Date of current version December 7, 2015. The Associate Editor coordinating the review process was Dr. Niclas Bjorsell.

The authors are with the Instrumentation, Sensors and Interfaces Group, Universitat Politècnica de Catalunya, Barcelona 08860, Spain (e-mail: jaime.oscar.casas@upc.edu).

Color versions of one or more of the figures in this paper are available online at http://ieeexplore.ieee.org.

Digital Object Identifier 10.1109/TIM.2015.2479105 amplitude-to-time conversion circuit [5] or, as $\mu \mathrm{C}$ manufacturers suggest, to use direct interface circuits (DICs) [6], [7]. Such circuits do not use an ADC and need only a capacitor, a reference resistance, and a $\mu \mathrm{C}$ that provides few digital ports and a timer. Because these are in little need of additional components, DICs could be interesting to reduce both the cost and the number of components of the measurement system [8]-[11]. Unfortunately, their use could also result in a higher energy consumption compared with conventional interface circuits that would lead to a reduction in the autonomy or an increase in the cost of the energy harvester system. Hence, a thorough analysis of the energy consumption of DICs is necessary in order to determine its suitability in energyconstrained systems.

The current consumed by a DIC was reported in [12] by analyzing the supply current waveform in each step of the measurement process, but it was not related to the measurement uncertainty specifications and the $\mu \mathrm{C}$ performance parameters. Some design guidelines that help in reducing consumption can be deduced from that analysis, but no information is given about its possible side effects on the measurement performance. This paper analyzes the energy consumption of DICs of resistive sensors and identifies the energy consumption sources to determine their dependence on the design specifications and parameters. A design tradeoff is obtained between the required measurement uncertainty and the resulting energy consumption. Considering this design constraint, a design guide is proposed for the selection of the adequate $\mu \mathrm{C}$, timer frequency, reference resistance, capacitance, or digital ports.

\section{BACKGROUND OPERATING PRINCIPLE}

Fig. 1 shows a basic DIC used to measure a resistive sensor $\left(R_{m}\right)$. The resistance $R_{m}$ is estimated by the discharging time $\left(T_{m}\right)$ of a capacitor $\left(C_{c}\right)$ through $R_{m}$. The output digital port PX.3 of a $\mu \mathrm{C}$ is held to $0 \mathrm{~V}\left(0\right.$ logic) and discharges $C_{c}$ from the supply voltage $\left(V_{\mathrm{DD}}\right)$ to the low threshold voltage limit $\left(V_{\mathrm{TL}}\right)$ of a digital Schmitt trigger input port (PX.0). The $\mu \mathrm{C}$ measures $T_{m}$ using an embedded timer whose frequency is $f_{\mathrm{CLK}}$ and calculates $R_{m}$ from

$$
R_{m}=\frac{T_{m}}{C_{c} \cdot \ln \left(\frac{V_{\mathrm{DD}}}{V_{\mathrm{TL}}}\right)}-R_{\mathrm{pm}}
$$

where $R_{\mathrm{pm}}$ is the output resistance of PX.3.

Unfortunately, using (1) to estimate $R_{m}$ results in a low accuracy due to the relatively high tolerance and temperature drift of $C_{c}, R_{\mathrm{pm}}$, and $V_{\mathrm{TL}}$. Fig. 2 shows a more complex 


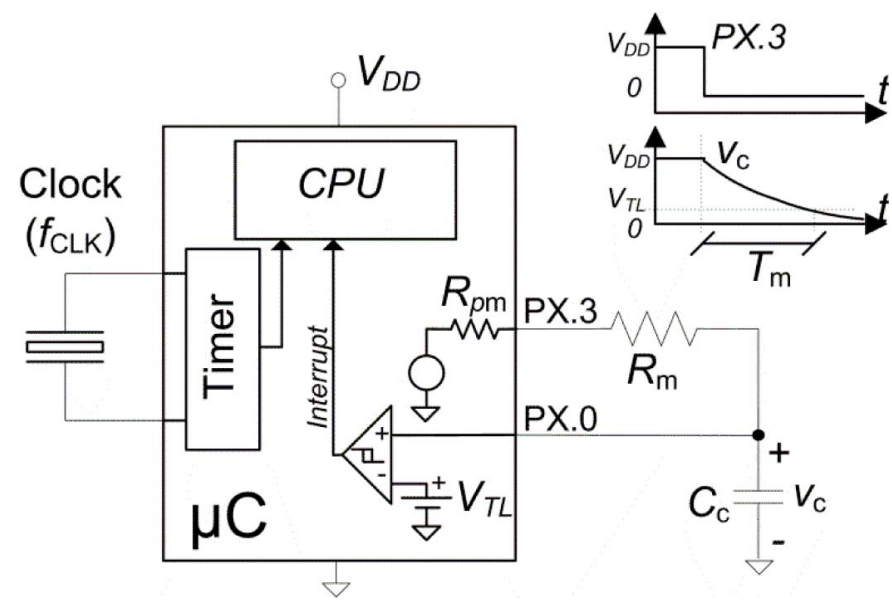

Fig. 1. Basic DIC of a resistive sensor $\left(R_{m}\right)$.

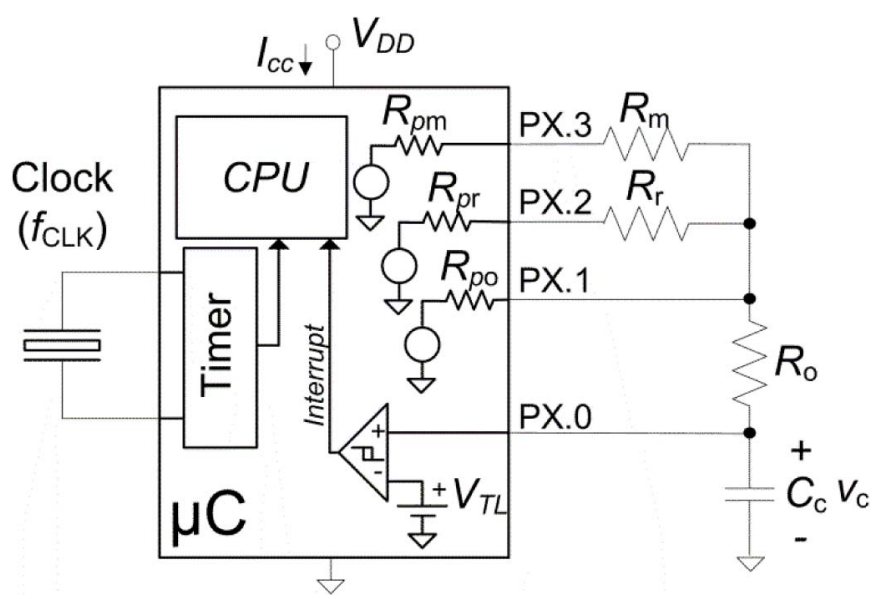

Fig. 2. DIC of a resistive sensor $R_{m}$ applying the three-signal measurement method [9].

circuit devised to achieve a higher accuracy [13], [14] where two digital ports (PX.2 and PX.1), a reference resistance $R_{r}$, and an offset resistance $R_{o}$ are added to the basic circuit.

Now, the time lengths of three discharge cycles of $C_{c}$ from $V_{\mathrm{DD}}$ to $V_{\mathrm{TL}}$ are measured, yielding $T_{m}, T_{r}$, and $T_{o}$ as shown in Fig. 3. There, one of the output ports is held to $0 \mathrm{~V}(0$ logic), while the others are held in high impedance. Previously to each discharge cycle, the capacitor is charged to the supply voltage $\left(V_{\mathrm{DD}}\right)$. The measurement process is performed in a very short time interval; hence, the temperature drift is negligible and the values of $R_{m}, R_{r}, R_{o}, C_{c}$, and $V_{\mathrm{TL}}$ can be considered constant during the three discharge cycles.

The relation between $R_{m}$ and the resulting discharging times is

$$
\begin{aligned}
& T_{m}=\left(R_{m}+R_{o}+R_{\mathrm{pm}}\right) \cdot C_{c} \cdot \ln \left(\frac{V_{\mathrm{DD}}}{V_{\mathrm{TL}}}\right) \\
& T_{r}=\left(R_{r}+R_{o}+R_{\mathrm{pr}}\right) \cdot C_{c} \cdot \ln \left(\frac{V_{\mathrm{DD}}}{V_{\mathrm{TL}}}\right) \\
& T_{o}=\left(R_{o}+R_{\mathrm{po}}\right) \cdot C_{c} \cdot \ln \left(\frac{V_{\mathrm{DD}}}{V_{\mathrm{TL}}}\right)
\end{aligned}
$$
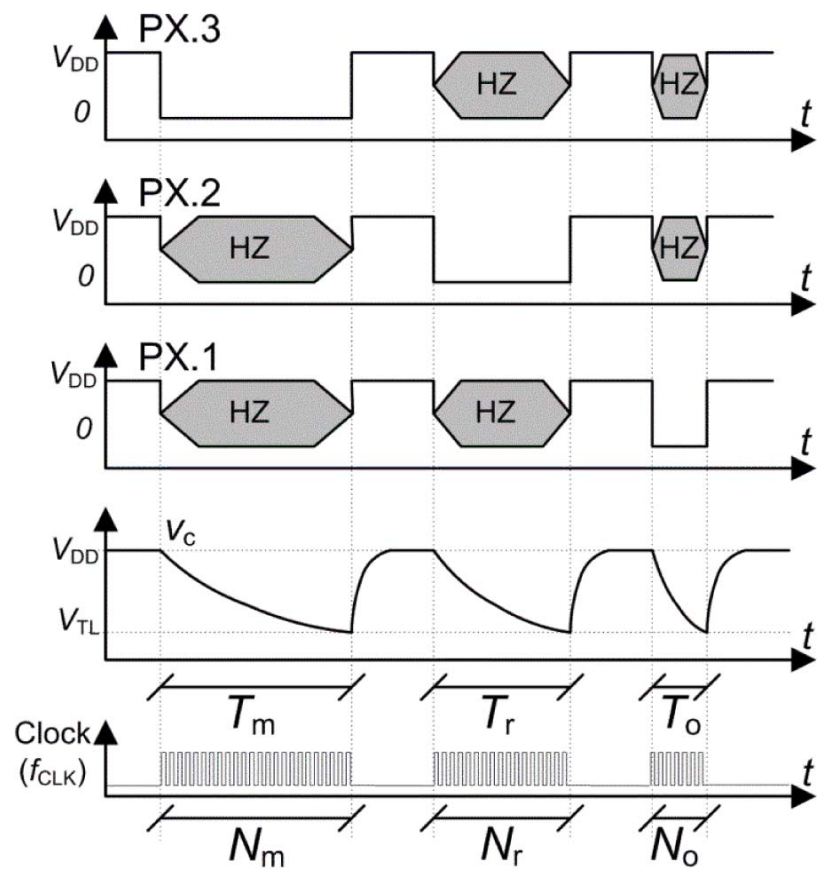

Fig. 3. Waveforms of the voltage in the $\mu$ C ports PX.3, PX.2, PX.1, and PX.0 during a measurement cycle.

where $R_{\mathrm{pm}}, R_{\mathrm{pr}}$, and $R_{\mathrm{po}}$ are the output resistances of the ports PX.3, PX.2, and PX.1, respectively.

Taking into account the symmetry of the output ports, we might approximate $R_{p} \approx R_{\mathrm{pm}} \approx R_{\mathrm{pr}} \approx R_{\mathrm{po}}$ and the sensor resistance can be calculated as

$$
R_{m}=R_{r} \cdot \frac{T_{m}-T_{o}}{T_{r}-T_{o}} \approx R_{r} \cdot \frac{N_{m}-N_{o}}{N_{r}-N_{o}}
$$

where $N_{m}, N_{r}$, and $N_{o}$ are the number of clock cycles during $T_{m}, T_{r}$, and $T_{o}$, respectively. If we neglect the timer quantization error, the time lengths $T_{j}$ can be approximated by $N_{j} / f_{\text {CLK }}$ (for $j=m, r$, or $o$ ). Note that (3) does not depend on $C_{c}, R_{p}$ and $V_{\mathrm{TL}}$, and hence, the accuracy in measuring $R_{m}$ is not limited by their tolerances nor their temperature drifts. Other limiting factors and their impact on the system performances have been analyzed in [15], where suggestions can also be found to improve the system performances.

\section{Design Constraints}

The existence of a tradeoff between the measurement uncertainty $\left(u_{R_{m}}\right)$ and the energy consumption $\left(E_{\text {Meas }}\right)$ will be demonstrated for the circuit of Fig. 2.

\section{A. Measurement Uncertainty}

$u_{R_{m}}$ is limited by the reference resistance uncertainty $\left(u_{R_{r}}\right)$ and the uncertainties of $N_{m}, N_{r}$, and $N_{o}\left(u_{N_{m}}, u_{N_{r}}\right.$ and $\left.u_{N_{o}}\right)$. Applying the law of uncertainty propagation [16], $u_{R_{m}}$ can be obtained from

$$
\begin{aligned}
& u_{R_{m}} \\
& =\sqrt{\left(\frac{\partial R_{m}}{\partial N_{m}}\right)^{2} u_{N_{m}}^{2}+\left(\frac{\partial R_{m}}{\partial N_{r}}\right)^{2} u_{N_{r}}^{2}+\left(\frac{\partial R_{m}}{\partial N_{o}}\right)^{2} u_{N_{o}}^{2}+\left(\frac{\partial R_{m}}{\partial R_{r}}\right)^{2} u_{R_{r}}^{2} .}
\end{aligned}
$$




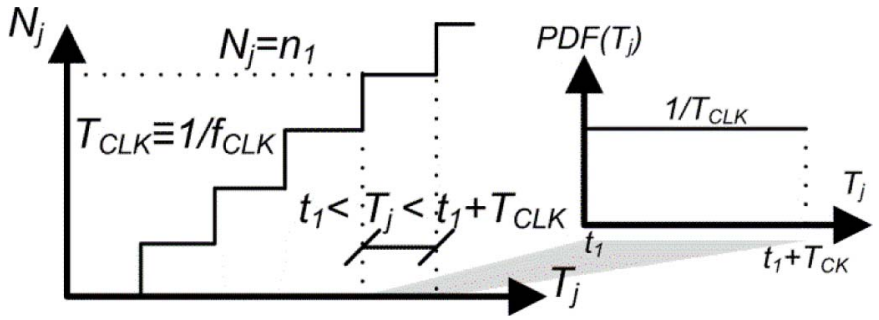

Fig. 4. Left: relationship between the timer count $N_{j}$ and the measured time $T_{j}$ when the quantization effects are considered alone. Right: the quantization error PDF of $T_{j}$ when $N_{j}=n_{1}$.

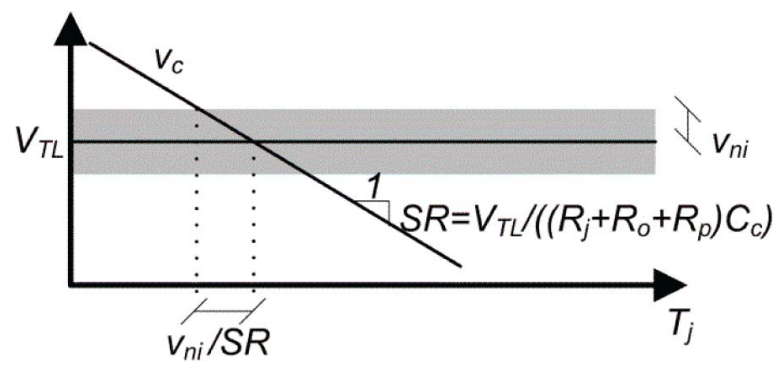

Fig. 5. Effect of the internal comparator trigger noise $\left(v_{\text {ni }}\right)$ and the signal SR on the measured time $\left(T_{j}\right)$.

Assuming $N_{j} \approx T_{j} f_{\mathrm{CLK}}$ (for $j=m, r$, or $o$ ), (2) and (3) are used to deduce

$$
\begin{aligned}
& \frac{\partial R_{m}}{\partial N_{m}} \approx \frac{1}{f_{\mathrm{CLK}} C_{C} \ln \left(V_{\mathrm{DD}} / V_{\mathrm{TL}}\right)} \\
& \frac{\partial R_{m}}{\partial N_{r}} \approx \frac{-1}{f_{\mathrm{CLK}} C_{C} \ln \left(V_{\mathrm{DD}} / V_{\mathrm{TL}}\right)} \cdot \frac{R_{m}}{R_{r}} \\
& \frac{\partial R_{m}}{\partial N_{o}} \approx \frac{R_{m} / R_{r}-1}{f_{\mathrm{CLK}} C_{C} \ln \left(V_{\mathrm{DD}} / V_{\mathrm{TL}}\right)} \\
& \frac{\partial R_{m}}{\partial R_{r}} \approx \frac{R_{m}}{R_{r}}
\end{aligned}
$$

and all uncertainties $u_{N_{j}}$ (for $j=m, r$, or $o$ ) appearing in (4) depend on the timer quantization uncertainty $\left(u_{N_{j} Q}\right)$, the comparator trigger uncertainty $\left(u_{N_{j} N}\right)$, and the uncertainties of the output port resistances $\left(u_{N_{j} R_{p}}\right)$. Applying again the law of uncertainty propagation, the overall uncertainty for $u_{N_{j}}(j=m, r$, or $o)$ is

$$
u_{N_{j}}=\sqrt{u_{N_{j} Q}^{2}+u_{N_{j} N}^{2}+u_{N_{j} R_{p j}}^{2}} .
$$

Fig. 4 shows the quantization effect and the error probability density function (PDF) of $T_{j}$. Assuming a rectangular density function [8], the uncertainty of $T_{j}$ will be $T_{\mathrm{CLK}} / \sqrt{12}$ and

$$
u_{N_{j} Q}=1 /\left(\sqrt{12} f_{\mathrm{CLK}}\right) \cdot f_{\mathrm{CLK}}=1 / \sqrt{12} .
$$

$u_{N_{j} N}$ is caused by the noise $\left(v_{\mathrm{ni}}\right)$ superimposed onto $V_{\mathrm{TL}}$ and the noise $\left(v_{\text {no }}\right)$ superimposed onto $v_{c}$. Fig. 5 shows that whenever $v_{c}$ crosses $V_{\mathrm{TL}}$, the noise $v_{\text {ni }}$ (and also $v_{\text {no }}$ ) result in an uncertainty of $T_{j}$ that depend on the slew rate (SR) of $v_{c}$. Considering the effect of $v_{\text {ni }}$ and $v_{\text {no }}$ at once and the SR,
$u_{N_{j} N}$ can be approximated by [14] as

$$
\begin{aligned}
& u_{N_{j} N} \approx \frac{\sqrt{v_{\mathrm{ni}}^{2}+v_{\mathrm{no}}^{2}}}{V_{\mathrm{TL}} /\left(\left(R_{j}+R_{o}+R_{p}\right) C_{c}\right)} f_{\mathrm{CLK}} j=m \text { or } n \\
& u_{N_{o} N} \approx \frac{\sqrt{v_{\mathrm{ni}}^{2}+v_{\mathrm{no}}^{2}}}{V_{\mathrm{TL} /\left(\left(R_{o}+R_{p}\right) C_{c}\right)}} f_{\mathrm{CLK}} .
\end{aligned}
$$

The propagation of the uncertainty, due to the digital port output resistance $\left(u_{R_{p}}\right)$, on the measured $N_{j}$ can be deduced from (2)

$$
u_{N_{j} R_{p}}=\frac{\partial T_{j}}{\partial R_{p}} f_{\mathrm{CLK}} u_{R_{p}}=f_{\mathrm{CLK}} C_{c} \ln \left(V_{\mathrm{DD}} / V_{\mathrm{TL}}\right) u_{R_{p}} .
$$

Finally, substituting (5)-(9) in (4), the overall uncertainty can be written as

$$
u_{R_{m}}=\sqrt{u_{R_{m} Q}^{2}+u_{R_{m} N}^{2}+u_{R_{m} R_{p}}^{2}+u_{R_{m} R_{r}}^{2}}
$$

where $u_{R_{m} Q}, u_{R_{m} N}, u_{R_{m} R_{p}}$, and $u_{R_{m} R_{r}}$ now group together the uncertainty terms due to the timer quantization, the comparator triggering, the output port resistance, and the reference resistor, respectively. Assuming that $R_{p}, R_{o} \ll R_{m}, R_{r}$, those uncertainties can be approximated to

$$
\begin{aligned}
& u_{R_{m} Q}^{2} \approx \frac{1+\left(\frac{R_{m}}{R_{r}}\right)^{2}+\left(\frac{R_{m}}{R_{r}}-1\right)^{2}}{\left(\sqrt{12} f_{\mathrm{CLK}} C_{C} \ln \left(V_{\mathrm{DD}} / V_{\mathrm{TL}}\right)\right)^{2}} \\
& u_{R_{m} N}^{2} \approx 2\left(\frac{R_{m} \sqrt{v_{\mathrm{ni}}^{2}+v_{\mathrm{no}}^{2}}}{V_{\mathrm{TL}} \ln \left(V_{\mathrm{DD}} / V_{\mathrm{TL}}\right)}\right)^{2} \\
& u_{R_{m} R_{p}}^{2} \approx 2\left(\left(\frac{R_{m}}{R_{r}}\right)^{2}-\frac{R_{m}}{R_{r}}+1\right)\left(u_{R_{p}}\right)^{2} \\
& u_{R_{m} R_{r}}^{2} \approx\left(\frac{R_{m}}{R_{r}}\right)^{2} u_{R_{r}}^{2} .
\end{aligned}
$$

Note that $u_{R_{m} Q}$ is inversely proportional to $f_{\mathrm{CLK}} C_{c}$, but $u_{R_{m} N}, u_{R_{m} R_{p}}$ and $u_{R_{m} R_{r}}$ neither depends on $f_{\mathrm{CLK}}$ nor $C_{c}$.

\section{B. Energy Consumption Model}

The energy consumed during a measurement cycle ( $\left.E_{\text {Meas }}\right)$ includes the charge $Q_{(3)}$ consumed by the control processing unit (CPU) to process the measurement algorithm based on (3), the charge consumed when charging $C_{c}$ from $V_{\mathrm{TL}}$ to $V_{\mathrm{DD}}$, the clock (Timer) charge consumption during $T_{m}, T_{r}$, and $T_{o}$, and the charge consumption caused by each Schmitt trigger input voltage transition $\left(Q_{H j}, j=m, n\right.$, or $o$ ) as follows:

$$
\begin{aligned}
E_{\text {Meas }}= & V_{\mathrm{DD}} Q_{(3)}+3 V_{\mathrm{DD}} C_{c} \cdot\left(V_{\mathrm{DD}}-V_{\mathrm{TL}}\right) \\
& +V_{\mathrm{DD}} I_{f_{\mathrm{CLK}}} \cdot\left(T_{m}+T_{r}+T_{o}\right)+V_{\mathrm{DD}} \\
& \cdot\left(Q_{\mathrm{Hm}}+Q_{\mathrm{Hr}}+Q_{\mathrm{Ho}}\right)
\end{aligned}
$$

where $I_{f_{\mathrm{CLK}}}$ stands for both the clock and the timer current consumption that depends on the clock frequency. The experimental data for $I_{f_{\mathrm{CLK}}}$ are shown in Fig. 6 where a linear 


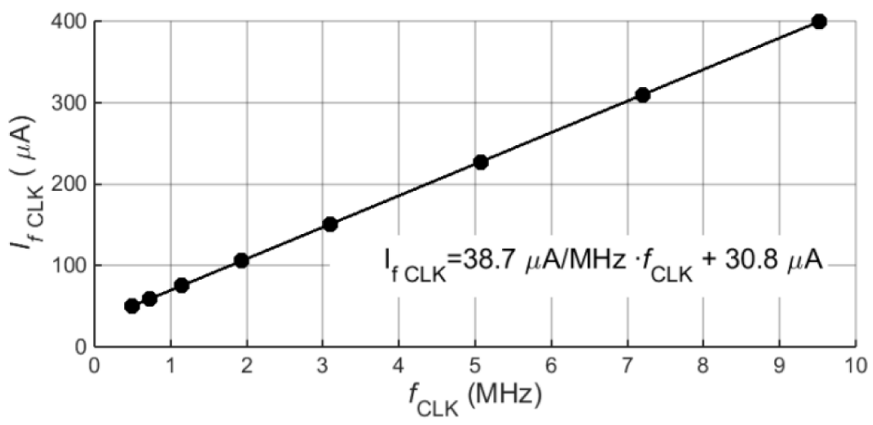

Fig. 6. Measured current consumption of the DCO clock and Timer B for the MSP430F1471.

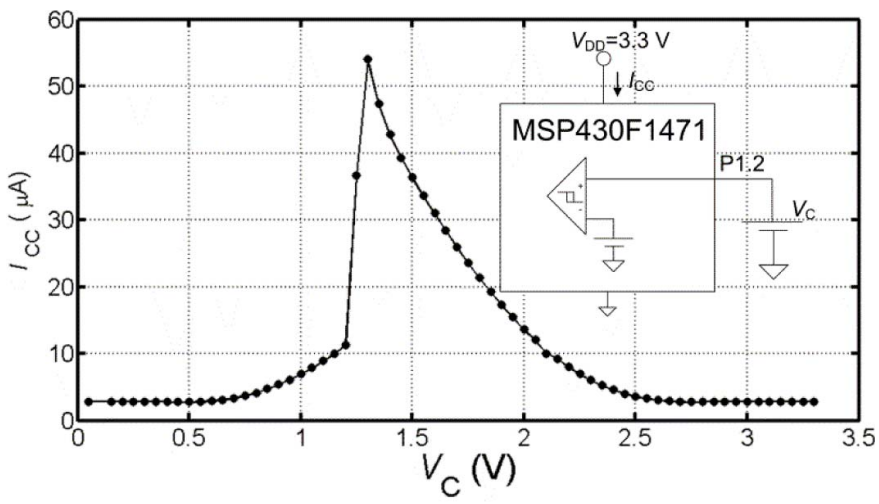

Fig. 7. Measured supply current of the MSP430F1471 versus the Schmitt trigger input voltage during a discharge cycle.

relationship between $I_{f_{\mathrm{CLK}}}$ and $f_{\mathrm{CLK}}$ is found for a commercial low-power $\mu \mathrm{C}$ (MSP430F1471). This current consumption will be modeled as $I_{f_{\mathrm{CLK}}} \approx k_{f} \cdot f_{\mathrm{CLK}}+k_{0}$ (the parameter values are shown in Fig. 6).

The processing charge $Q_{(3)}$ depends only on the number of instructions used by the CPU to process the measurement and not on the CPU clock frequency [17]. Hence, the processing code must be optimized to minimize it. The $\mu \mathrm{C}$ supply current increases as the input voltage of the Schmitt trigger gets closer to the threshold voltage limit $V_{\mathrm{TL}}$ [18]. Fig. 7 shows the experimental data for the MSP430F1471 supply current when the voltage falls from 3.3 to $0 \mathrm{~V}$. It can be concluded that the charge consumed by the Schmitt trigger is smaller as shorter is the voltage transition time and that it can be dismissed for fast voltage transients, such as the charge cycle of $C_{c}$. In the case of the discharge cycles, the charge consumption is given by

$$
\begin{aligned}
Q_{H j} & =\int_{T_{j}}\left(I_{\mathrm{cc}}-I_{\mathrm{co}}\right) d t \\
& = \begin{cases}\left(R_{j}+R_{p}+R_{o}\right) C_{c} I_{H} & j=m \text { and } r \\
\left(R_{p}+R_{o}\right) C_{c} I_{H} & j=o\end{cases}
\end{aligned}
$$

where the time integration was changed to a voltage integration using the known capacitor voltage $\left(v_{c}\right)$ time dependence during the discharge cycles. The value of $I_{H}$ is calculated

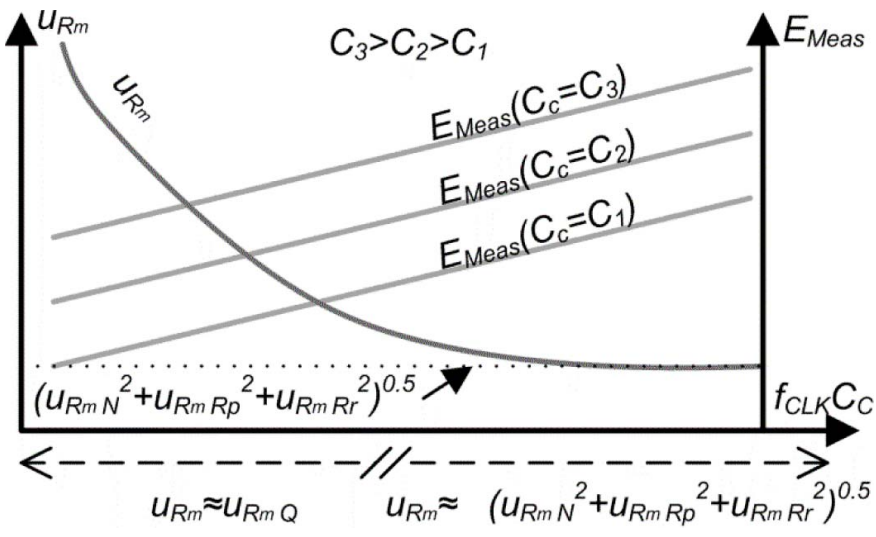

Fig. 8. $\quad E_{\text {Meas }}$ and $u_{R_{m}}$ versus $f_{\mathrm{CLK}} C_{C}$ for several values of $C_{C}$. Demonstration of the tradeoff between $E_{\text {Meas }}$ and $u_{R_{m}}$ when $f_{\mathrm{CLK}} C_{C}$ is selected.

from the data shown in Fig. 7 using

$$
I_{H} \equiv \int_{V_{\mathrm{TL}}}^{V_{\mathrm{DD}}} \frac{\left(I_{\mathrm{cc}}-I_{\mathrm{co}}\right)}{v_{c}} d v_{c}
$$

where $I_{\mathrm{co}}$ is the supply current when the Schmitt trigger input is at the rails and is also obtained from Fig. 7.

Substituting (13) and (14) in (12), $E_{\text {Meas }}$ can be approximated to

$$
\begin{aligned}
E_{\text {Meas }} \approx & V_{\mathrm{DD}}\left(R_{m}+R_{r}+3 R_{o}+3 R_{p}\right) \ln \left(\frac{V_{\mathrm{DD}}}{V_{\mathrm{TL}}}\right) k_{f} f_{\mathrm{CLK}} C_{C} \\
& +V_{\mathrm{DD}}\left(Q_{(3)}+3 C_{c} \cdot\left(V_{\mathrm{DD}}-V_{\mathrm{TL}}\right)\right) \\
& +V_{\mathrm{DD}}\left(R_{m}+R_{r}+3 R_{o}+3 R_{p}\right) C_{C} \\
& \cdot\left(\ln \left(\frac{V_{\mathrm{DD}}}{V_{\mathrm{TL}}}\right) k_{0}+I_{H}\right)
\end{aligned}
$$

which increases with $f_{\mathrm{CLK}} C_{C}$ or $C_{C}$. Fig. 8 shows the dependence of $u_{R_{m}}$ and $E_{\text {Meas }}$ on $f_{\mathrm{CLK}} C_{C}$ according to their mathematical descriptions and demostrates that a tradeoff exists between the measurement uncertainty and the energy consumption: while the uncertainty can be improved by increasing the value of $f_{\mathrm{CLK}} C_{C}$, this also implies an increase of the energy consumption.

Fig. 8 also shows that the uncertainty eventually reaches a lower bound for large values of $f_{\mathrm{CLK}} C_{C}$ where the errors introduced by the trigger noise and the resistance tolerance become dominant over those associated with the timer. On the other hand, the uncertainty increases for the smaller values of $f_{\mathrm{CLK}} C_{C}$ where the quantization uncertainty is dominant. Therefore, a low-power DIC design should work on the lowest possible range for $f_{\mathrm{CLK}} C_{C}$, because outside of this region, an increase in $f_{\mathrm{CLK}} C_{C}$ steps up the energy consumption without a noteable improvement on $u_{R_{m}}$.

\section{Proposed Design Procedure}

For designs comprising the following set of specifications, we look forward to finding the proper values of $R_{r}, R_{o}, C_{c}$, and $f_{\mathrm{CLK}}$ that minimizes the $E_{\text {Meas }}$ :

1) a maximum allowed uncertainty $u_{R_{m} \operatorname{Max}}$;

2) a given $\mu \mathrm{C}$ setup (fixed $V_{\mathrm{DD}}$ and $V_{\mathrm{TL}}$ );

3) a measurement range $R_{\min }<R_{m}<R_{\text {Max }}$. 


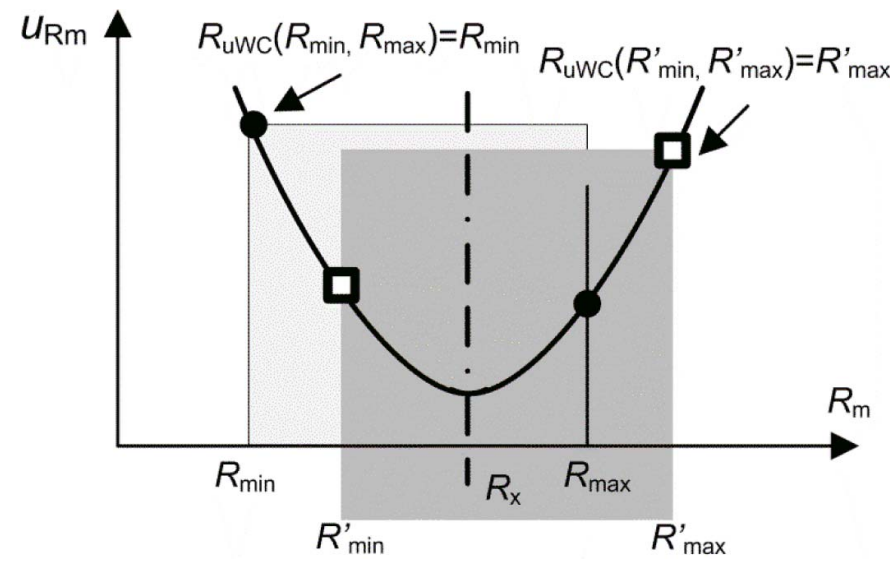

Fig. 9. Evaluation of $R_{u \mathrm{WC}}$ in two scenarios. $R_{u \mathrm{WC}}=R_{\min }$ for a measurement range $\left(R_{\min }, R_{\max }\right)$ and $R_{u \mathrm{WC}}=R_{\max }^{\prime}$ for $\left(R_{\min }^{\prime}, R_{\max }^{\prime}\right)$.

Because both uncertainty and measurement energy also depend on $R_{m}$, we might run into two adverse scenarios in the measurement range, a value of $R_{m}$ for which the uncertainty is maximum $\left(R_{u \mathrm{WC}}\right)$, and a value for which the energy consumption is maximum $\left(R_{E \mathrm{WC}}\right)$. The uncertainty limits must held for $R_{m}=R_{u \text { WC, }}$, while the energy consumption must be minimized for $R_{m}=R_{E \mathrm{WC}}$. Assuming that usually $R_{p} \ll R_{m}$ and a high-precision resistance will be selected for $R_{r}$, we dismiss $u_{R_{m} R_{p}}$ and $u_{R_{m} R_{r}}$ in this procedure. We propose the following design steps.

Step 1: Selection of $R_{o}$. Equations (6)-(11) and (15) show that the uncertainty and energy consumption decrease to $R_{o}$. Therefore, we propose to select the minimum value of $R_{O}$ determined by the maximum output current sourced by PX.1.

Step 2: Selection of $f_{\mathrm{CLK}}$. On one hand, $E_{\text {Meas }}$ is proportional to $C_{C}$ for a given $f_{\mathrm{CLK}} C_{C}$ value. On the other hand, a minimum value of $f_{\mathrm{CLK}} C_{C},\left.f_{\mathrm{CLK}} C_{C}\right|_{\mathrm{Min}}$, is required to achieve an uncertainty compliant with the design specifications. Hence, $f_{\text {CLK }}$ should be selected as the maximum frequency $f_{\text {CLKMax }}$ supported by the $\mu \mathrm{C}$. In this way, $C_{C}$ and also $E_{\text {Meas }}$ will be minimum $\left(C_{C \text { min }}=\left.f_{\mathrm{CLK}} C_{C}\right|_{\text {Min }} / f_{\mathrm{CLKMax}}\right)$.

Step 3: Selection of $R_{r}$. The optimum value is selected to achieve minimum $E_{\text {Meas }}$ for $R_{E \mathrm{WC}}$, while holding $u_{R_{m \mathrm{Max}}}$ for $R_{u \mathrm{WC}}$.

$E_{\text {Meas }}\left(R_{m}\right)$ in (15) is a continuously increasing function and hence $R_{E \mathrm{WC}}=R_{\text {Max }}$. On the other hand, $u_{R_{m}}\left(R_{m}\right)$ is a polynomial of second degree in (10) with a minimum at $R_{x}$. Taking into account the symmetry of $u_{R_{m}}\left(R_{m}\right)$ with respect to $R_{x}$ (Fig. 9), $R_{u \mathrm{WC}}$ is given by

$$
R_{u \mathrm{WC}}= \begin{cases}R_{\mathrm{Max}} & \text { if } R_{x} \leq \frac{R_{\mathrm{Max}}+R_{\mathrm{min}}}{2} \\ R_{\min } & \text { if } R_{x}>\frac{R_{\mathrm{Max}}+R_{\mathrm{min}}}{2}\end{cases}
$$

The value of $R_{x}$ is obtained by equating to zero the first derivative of $u_{R_{m}}\left(R_{m}\right)$ in (10). The resulting $R_{x}$ depends on the sizes of $C_{C}$ and $R_{r}$ that have not yet been selected. Using this expression in (16) and considering the value for $C_{C}$ that yields $u_{R_{m} \text { Max }}$ in (10) and (11) when $R_{m}=R_{u \text { WC }}=R_{\min }$, we rewrite (16) as

$$
R_{u \mathrm{WC}}= \begin{cases}R_{\mathrm{Max}} & \text { if } R_{r} \leq R_{\gamma} \\ R_{\min } & \text { if } R_{r}>R_{\gamma}\end{cases}
$$

where

$$
R_{\gamma} \equiv \frac{\left(R_{\mathrm{Max}}+R_{\mathrm{min}}\right)}{\gamma}\left(1-\sqrt{1-\frac{2 \gamma}{1+2 \frac{\left(v_{\mathrm{ni}}^{2}+v_{\mathrm{no}}^{2}\right) R_{\mathrm{Max}} R_{\min }}{\left(u_{R_{m} \mathrm{Max}} V_{\mathrm{TL}} \ln \left(\frac{V_{\mathrm{DD}}}{v_{\mathrm{TL}}}\right)\right)^{2}}}}\right)
$$

and

$$
\gamma \equiv \frac{4\left(v_{\mathrm{ni}}^{2}+v_{\mathrm{no}}^{2}\right)\left(R_{\mathrm{Max}}+R_{\mathrm{min}}\right)^{2}}{\left(u_{R_{m} \mathrm{Max}} V_{\mathrm{TL}} \ln \left(\frac{V_{\mathrm{DD}}}{V_{\mathrm{TL}}}\right)\right)^{2}+2\left(v_{\mathrm{ni}}^{2}+v_{\mathrm{no}}^{2}\right) R_{\mathrm{Max}} R_{\min }} .
$$

$R_{\gamma}$ can be approximated by $R_{\mathrm{Max}}+R_{\min }$ when the noise voltage is negligible $\left(v_{\text {ni }}^{2}+v_{\text {no }}^{2} \approx 0\right.$ and $\left.\gamma \approx 0\right)$ and it will be higher otherwise. Whenever a negative number is in the square root of (18), the value of $R_{\gamma}$ must be considered as $\infty$.

Using these values for $R_{E \mathrm{WC}}$ and $R_{u \mathrm{WC}}$, and neglecting $R_{o}$, $R_{p}, u_{R_{m} R_{r}}$, and $u_{R_{m} R_{p}}$, the energy consumption is obtained from (10), (11a), and (15)

$$
\begin{aligned}
E_{\text {Meas }} \approx & V_{\mathrm{DD}} Q_{3}+\frac{V_{\mathrm{DD}} \cdot k_{f} \cdot R_{\mu}}{2 \sqrt{3} \sqrt{u_{R_{m \mathrm{Max}}}^{2}-u_{R_{m N}}^{2}}} \\
& \cdot \sqrt{1+\frac{R_{u W C}^{2}}{R_{r}^{2}}+\left(\frac{R_{u \mathrm{WC}}}{R_{r}}-1\right)^{2}} \\
& \cdot\left(1+\frac{R_{\mathrm{Max}}+R_{r}}{R_{\mu}}\left(1+\alpha_{\mu}\right)\right)
\end{aligned}
$$

where $u_{R_{m N}}$ is that obtained for $R_{m}=R_{u \text { WC }}$ and

$$
\begin{aligned}
R_{\mu} & \equiv \frac{3\left(V_{\mathrm{DD}}-V_{\mathrm{TL}}\right)}{f_{\mathrm{CKMax}} \cdot k_{f} \cdot \ln \left(V_{\mathrm{DD}} / V_{\mathrm{TL}}\right)} \\
\alpha_{\mu} & \equiv \frac{\ln \left(\frac{V_{\mathrm{DD}}}{V_{\mathrm{TL}}}\right) k_{0}+I_{H}}{f_{\mathrm{CLKMax}} \cdot k_{f} \cdot \ln \left(V_{\mathrm{DD}} / V_{\mathrm{TL}}\right)} .
\end{aligned}
$$

Note that $R_{\mu}$ and $\alpha_{\mu}$ depend only on the $\mu \mathrm{C}$ performance parameters. On the other hand, the value of $R_{\mu}$ sets limits for $R_{m}$ and $R_{r}$ that yield a negligible clock current consumption. Specifically, if $R_{\mathrm{Max}}+R_{r} \ll R_{\mu}$, the value of $E_{\text {Meas }}$ will not depend on $k_{f}$ or $k_{o}$.

Fig. 10 shows a qualitative representation of $E_{\text {Meas }}$ versus $R_{r}$. If $R_{r}$ is lower than $R_{\gamma}, E_{\text {Meas }}$ is given by (20) for $R_{u \mathrm{WC}}=R_{\mathrm{Max}}$. Otherwise, it must be assumed that $R_{u \text { WC }}=R_{\min }$. The resulting function does not have a discontinuity because they match at the intersection point.

$R_{\text {opt }}\left(R_{\mathrm{Max}}\right)$ and $R_{\mathrm{opt}}\left(R_{\min }\right)$ are, respectively, the values of $R_{r}$ at the minimum points of $E_{\text {Meas }}\left(R_{u \mathrm{WC}}=R_{\mathrm{Max}}\right)$ and $E_{\text {Meas }}\left(R_{u \mathrm{WC}}=R_{\min }\right) . R_{\mathrm{opt}}\left(R_{\mathrm{Max}}\right)$ can be higher or lower than $R_{\gamma}$, whereas $R_{\text {opt }}\left(R_{\min }\right)$ is always lower. Therefore, the minimum energy consumption is achieved for

$$
R_{r}=\min \left(R_{\mathrm{opt}}\left(R_{\mathrm{Max}}\right), R_{\gamma}\right)
$$




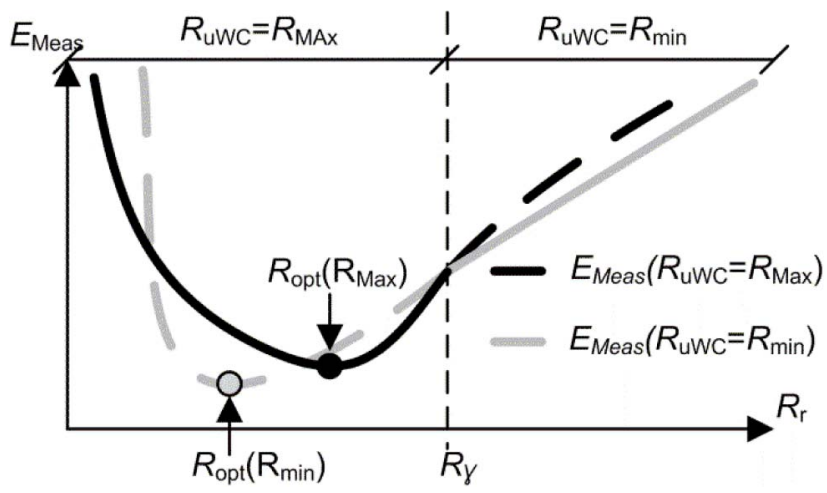

Fig. 10. Representation of $E_{\mathrm{Meas}}$ for $R_{u} \mathrm{WC}=R_{\mathrm{Max}}$ and $R_{u} \mathrm{WC}=R_{\min }$

where

$$
R_{\mathrm{opt}}\left(R_{\mathrm{Max}}\right) \cong R_{\mathrm{Max}} \frac{2 R_{\mu} / R_{\mathrm{Max}}+8\left(1+\alpha_{\mu}\right)}{R_{\mu} / R_{\mathrm{Max}}+8\left(1+\alpha_{\mu}\right)} .
$$

Note that the value of $R_{r}$ given in (22) always implies that $R_{u \mathrm{WC}}=R_{\mathrm{Max}}$.

Step 4: Selection of $C_{C}$. Once that $R_{r}$ has been chosen from the previous step 3 , use (10) and (11) to find $\left.\left(f_{\mathrm{CLK}} C_{C}\right)\right|_{\text {Min }}$ that satisfies the uncertainty requirements. The optimum capacitance is given by $\left.\left(f_{\mathrm{CLK}} C_{C}\right)\right|_{\mathrm{Min}} / f_{\mathrm{CLKMax}}$.

The minimum energy consumption for a specific uncertainty can thus be obtained by substituting the selected parameters in (20). Any attempts to further reduce $u_{R_{m}}$ Max will inevitably result in an increased energy consumption. That demonstrates again the tradeoff between them.

\section{Design AND EXPERIMENTAL Results}

We selected the MSP430F1471 (Texas Instruments, Inc.) to test the proposed energy consumption model and the design procedure. This low-power $\mu \mathrm{C}$ does not provide an $\mathrm{ADC}$ and the manufacturer recommends using a DIC to measure resistive elements [19]. It provides five low-power operating modes in which the CPU and the clocks can be dynamically disabled to reduce power consumption. It also provides a comparator (Comparator_A) that avoids the charge consumption caused by conventional Schmitt trigger digital inputs $\left(I_{h}=0\right)$. Nevertheless, because most low-power $\mu$ Cs do not provide it, its use was discarded in order to draw conclusions as general as possible. Instead, we chose four general-purpose Schmitt trigger digital ports to implement the method in [13] and [14] as described in Fig. 3: P1.2 is PX.0, and P2.1, P2.2, and P2.3 are PX.1, PX.2, and PX.3, respectively.

The 32-kHz low-power clock (ACLK) was used to control the length of the charge cycles and trigger the measurements, and the embedded high-frequency digitally controlled oscillator (DCO) [18] was used to measure $T_{m}, T_{r}$, and $T_{o}$ (Fig. 11). ACLK is always active and is the clock source for the watchdog timer producing an interrupt service that periodically calls the measurement routine once per second. It is also the Timer B clock source, which is responsible for producing an interrupt service each time a charge cycle is finished and when a new discharge cycle must be started.

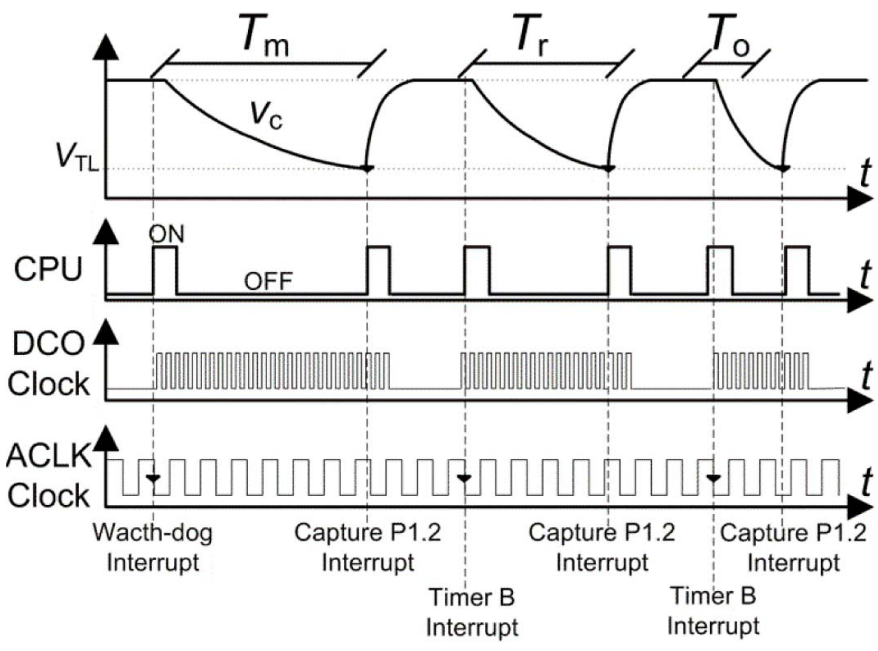

Fig. 11. Measurement timing diagram, showing the activity of the CPU, ACLK, and DCO.

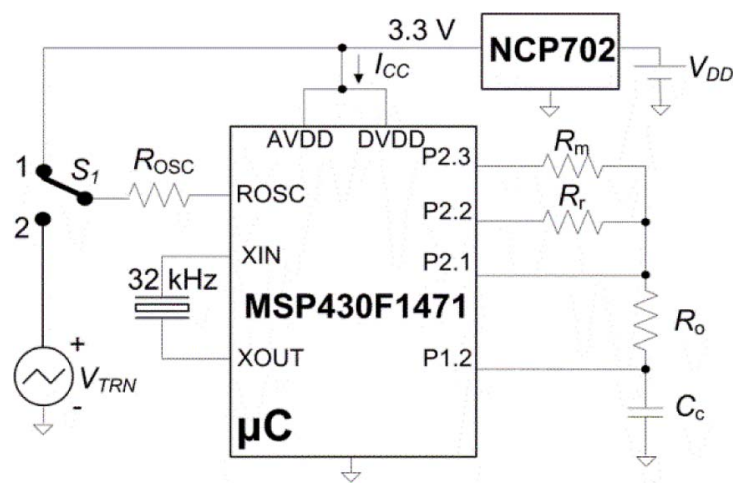

Fig. 12. $\mu \mathrm{C}$ experimental setup.

The DCO is an $R C$-type clock with fast startup and shutdown. It is the clock source for both the CPU and the timer A. The capture module of timer A triggers an interrupt when $v_{c}$ reaches $V_{\mathrm{TL}}$ and captures $N_{m}, N_{r}$, and $N_{o}$. The CPU and DCO are shutdown when they are not used in order to save energy.

A low-power 3.3 V LDO (NCP702, ON Semiconductor) was selected to supply the $\mu \mathrm{C}$ (Fig. 12) due to its low noise that results in a lower comparator trigger level noise [20]. This supply voltage defines $V_{\mathrm{TL}}=1.2 \mathrm{~V}$ and $f_{\mathrm{CLKMax}}=7 \mathrm{MHz}$ for the DCO clock [19]. Under these operating conditions, we obtained the data shown in Figs. 6 and 7, yielding $K_{f}=38.7 \mu \mathrm{A} / \mathrm{MHz}, K_{0}=30.8 \mu \mathrm{A}$, and $I_{h}=16.17 \mu \mathrm{A}$. These and all other measurements were performed using a 6(1/2) digital multimeter with 100 power-line cycles integration period (Agilent 34410A). Substituting these values in (21), we obtained $R_{\mu}=23 \mathrm{k} \Omega$ and $\alpha_{\mu}=0.17$.

A $100 \mathrm{k} \Omega$ external resistance $\left(R_{\mathrm{OSC}}\right)$ was used to reduce the temperature drift of the DCO frequency $\left(f_{\mathrm{CLK}}\right)$. Usually, this resistance is biased by the supply voltage to achieve a constant frequency $\left(S_{1} \rightarrow 1\right)$.

The proposed design procedure and energy consumption model (15) were validated for two different design scenarios. One of them uses a sensor resistance much lower than $R_{\mu}$ and the other uses a larger one. The first scenario is the 


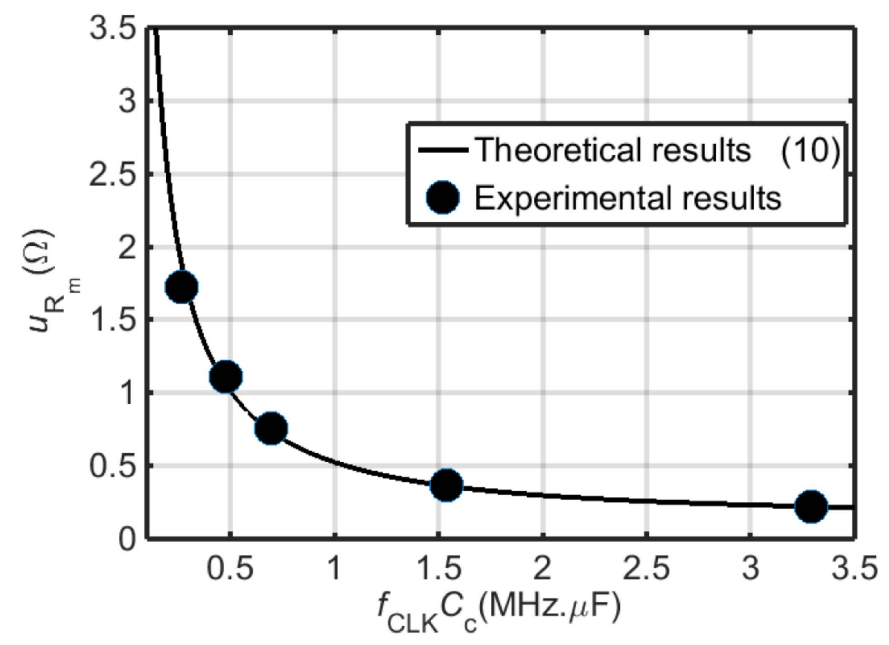

Fig. 13. $u_{R_{m}}$ versus $\left(f_{\mathrm{CLK}} C_{C}\right)$ for $R_{m}=1.38 \mathrm{k} \Omega, R_{r}=1 \mathrm{k} \Omega$, and $f_{\mathrm{CLK}}=7 \mathrm{MHz}$.

design of an interface circuit of a PT1000 sensor whose resistance ranges between $R_{\min }=1 \mathrm{k} \Omega$ and $R_{\mathrm{Max}}=1.38 \mathrm{k} \Omega$ corresponding to a temperature range from $0{ }^{\circ} \mathrm{C}$ and $100{ }^{\circ} \mathrm{C}$. Prior to setting an uncertainty specification, we measured $u_{R_{m}}$ to estimate $\left(v_{\mathrm{ni}}^{2}+v_{\mathrm{no}}^{2}\right)^{1 / 2}$ and compared the experimental results with (10). In fact, getting a complete knowledge of this noise would require getting real data from several $\mu \mathrm{Cs}$ configured with different resistances, capacitances, and temperatures that would let us observe the quantization effect for the measurement of a fixed $R_{m}$. Because this is not practical, we decided to introduce a controlled perturbation in the DCO clock frequency. During the measurement process, $R_{\mathrm{OSC}}$ was biased by a $0.2-\mathrm{Hz}$ triangular waveform voltage ( $\left.V_{\text {TRN }}\right)$ that varies between 3.3 and $3.2 \mathrm{~V}\left(S_{1} \rightarrow 2\right)$. This slow variation in the bias causes a small perturbation on $f_{\text {CLK }}$ (from 6.9 to $7.1 \mathrm{MHz}$ ) that emulates the effects of the tolerances and temperature drifts, letting us observe the effect of the quantization uncertainty.

The measurements were repeated four hundred times for each value of $C_{c}$. The use of such a low-frequency signal means that $f_{\text {CLK }}$ can be considered constant during a complete measuring cycle (changes below the resolution of the system). Fig. 13 shows the measured uncertainties for $R_{m}=R_{u \mathrm{WC}}=$ $1.38 \mathrm{k} \Omega, R_{r}=1 \mathrm{k} \Omega, f_{\text {CLK }} \sim 7 \mathrm{MHz}$, and several values of $C_{c}$ that ranged from 38 to $470 \mathrm{nF}$.

Fitting (10) to the experimental results allowed to deduce that $\left(v_{\mathrm{ni}}^{2}+v_{\mathrm{no}}^{2}\right)^{1 / 2}$ was about $101 \mu \mathrm{V}$, and consequently, $u_{R_{m} N}$ was $0.16 \Omega(11 \mathrm{~b})$. That delimits the maximum ENOB value at 10 bits, for the design specifications and to 12 bits if the measurement range is expanded from $0 \Omega$ to $R_{\mathrm{Max}}$. This limit does not depend on $R_{r}$ and depends only on the performance parameters of the $\mu \mathrm{C}$ and on the regulator voltage noise that provides the supply.

As a practical example, the use of a type B-PT1000 RTD sensor is presented. In the range of $0{ }^{\circ} \mathrm{C}-100{ }^{\circ} \mathrm{C}$ ( $1 \mathrm{k} \Omega$ to $1.38 \mathrm{k} \Omega$ ), the uncertainty of the sensor is $0.8{ }^{\circ} \mathrm{C}$ (resistor change about $3 \Omega$ ). To maintain the contribution of the conditioning circuit over three times below this error, the uncertainty of the direct interface design is specified at

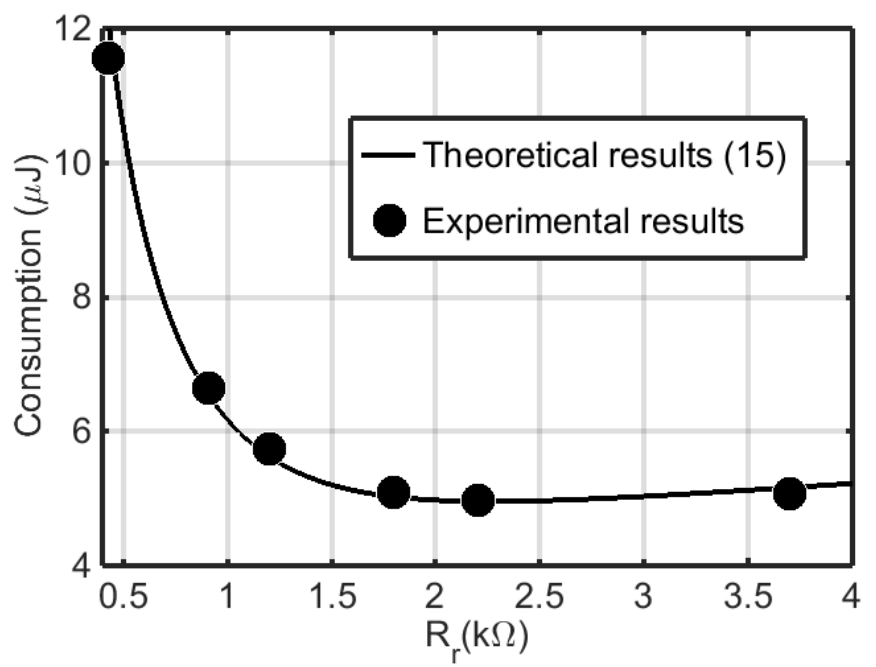

Fig. 14. Energy consumption versus $R_{r}$ for $R_{m}=1.38 \mathrm{k} \Omega$ and $u_{R_{m \text { Max }}}=0.38 \Omega$. The value of $C_{C}$ is modified for each value of $R_{r}$ to achieve the desired $u_{R_{m \mathrm{Max}}}$.

$u_{R_{m \text { Max }}}=0.38 \Omega(9 \mathrm{ENOB})$, which translates into an equivalent temperature error of the conditioning circuit of $\pm 0.2{ }^{\circ} \mathrm{C}$ for a $95 \%$ confidence interval. Following the design steps described above, a $130 \Omega$ resistor was selected for $R_{O}$ (step 1) and $7 \mathrm{MHz}$ for $f_{\text {CLK }}$ (step 2). Equations (22) and (10) were used to determine that the optimum values for $R_{r}$ and $C_{c}$ are, respectively, $2.27 \mathrm{k} \Omega$ and $144 \mathrm{nF}$ (steps 3 and 4, respectively). Fig. 14 shows that the energy consumption is minimum for these values and that the experimental results fit the theoretical results predicted by (15).

As the watch-dog timer period sets a periodical measuring cycle, the charge and energy consumption was measured from the average valued of $I_{\mathrm{cc}}$ (Fig. 12). For these measurements, the watch-dog timer period was changed to $15.625 \mathrm{~ms}$ to improve the measuring accuracy of $I_{\mathrm{cc}}$ and the switch S1 was connected to 1 . These measurements were also performed for $C_{c}=0 \mu \mathrm{F}$ to determine that $Q_{(3)}$ is $440 \mathrm{nC}(15)$.

The measurement setup was also used for a DIC with a measuring range one hundred times higher $\left(R_{\min }=100 \mathrm{k} \Omega\right.$ and $R_{\mathrm{Max}}=138 \mathrm{k} \Omega$ ). This new range would correspond to the design specifications of a conditioner circuit for a highly resistive sensor, such as a light-dependent resistor. In this scenario, we measured $u_{R_{m}}$ for $R_{u W C}=138 \mathrm{k} \Omega, R_{r}=100 \mathrm{k} \Omega$, and several capacitor sizes between $0.47 \mathrm{nF}$ and $4.7 \mathrm{nF}$. Fig. 15 shows the experimental results and the theoretical curve obtained for the best fitting value of $\left(v_{\mathrm{ni}}^{2}+v_{\mathrm{no}}^{2}\right)^{1 / 2}$, which in this case is equal $92 \mu \mathrm{V}$. This value is similar to the value achieved in the previous scenario.

The new design used an $u_{R m \operatorname{Max}}=38 \Omega$. In this case, the values of $R_{r}$ and $C_{c}$ obtained from the design procedure were, respectively, $140 \mathrm{k} \Omega$ and $16 \mathrm{nF}$. Fig. 16 shows that the energy consumption was also minimum for these specific design parameters.

Note that the consumption is hardly increased for values of $R_{r}$ higher than the optimum value in Fig. 14. Nevertheless, this variation is higher in Fig. 16. This increment is mainly caused by the DCO clock. In the first scenario, $R_{\text {Max }}$ is much 


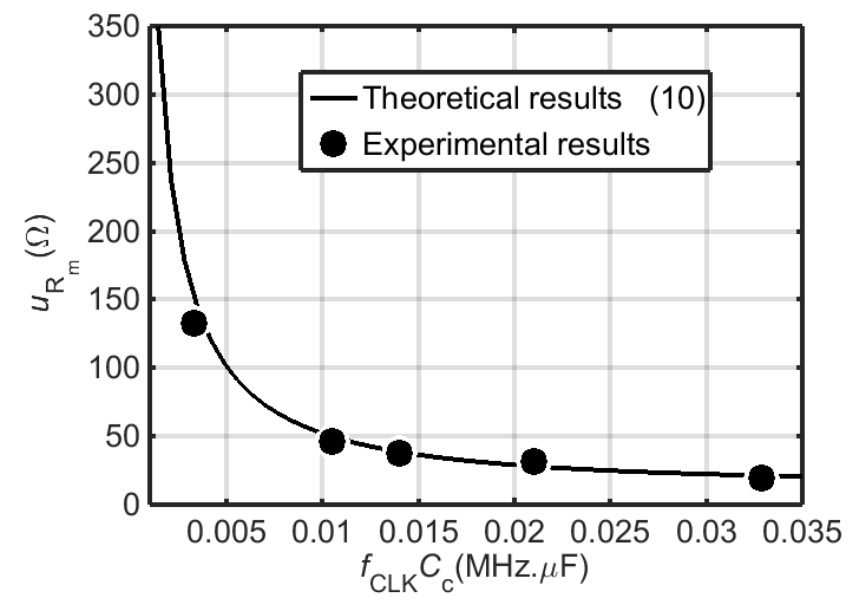

Fig. 15. $u_{R_{m}}$ versus $f_{\mathrm{ClK}} C_{C}$ for $R_{m}=138 \mathrm{k} \Omega, R_{r}=100 \mathrm{k} \Omega$, and $f_{\mathrm{ClK}}=7 \mathrm{MHz}$

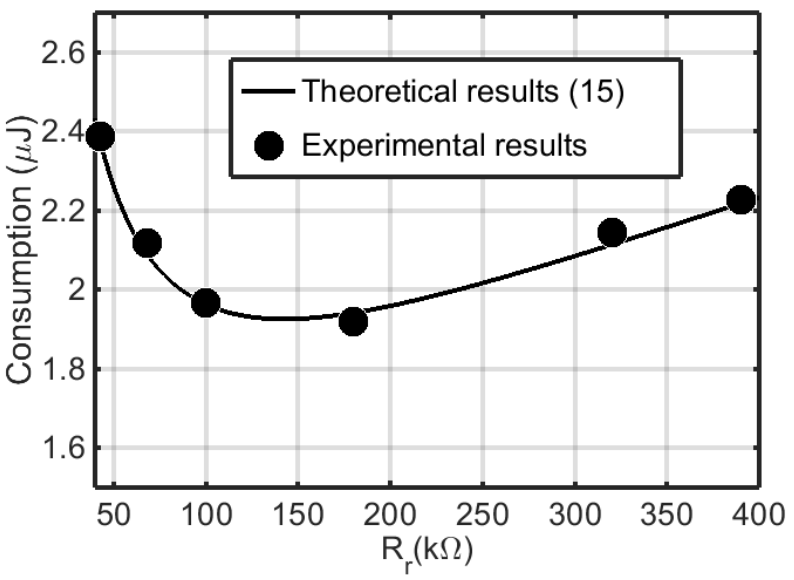

Fig. 16. Energy consumption versus $R_{r}$ for $R_{m}=138 \mathrm{k} \Omega$ and $u_{R_{m \text { Max }}}=$ $38 \Omega$. The value of $C_{C}$ was modified for each value of $R_{r}$ to achieve the desired $u_{R_{m \text { Max }}}$

lower than $R_{\mu}$, and as a consequence, the clock consumption is insignificant. The higher value selected for $R_{\mathrm{Max}}$ in the second scenario makes the consumption increase significantly.

\section{CONCLUSION}

This paper has analyzed the energy consumption of resistive DICs. The energy is used to charge the capacitor, to supply the clock timer and the Schmitt trigger input port and to process the measuring algorithm by the CPU. The higher the capacitor, the clock frequency, or the reference resistance, the higher is the accuracy but, unfortunately, also is the energy consumption.

To achieve minimum energy consumption for a given uncertainty, the clock frequency must be selected as high as possible, and an optimum value for the capacitor and for the reference resistor can be found. The values of this reference resistor below the optimum value imply a larger capacitor, and consequently, an increase of the capacitor charge. Conversely, higher values would imply measuring longer discharge cycles and hence a higher energy to supply the clock timer. The clock timer consumption is more significant the higher the maximum sensor resistance in the measuring range is in relation to a defined $\mu \mathrm{C}$ parameter $R_{\mu}$.

The optimum energy consumption for the design specifications is in the $\mu \mathrm{Jth}$ order of magnitude, and hence, it is much higher than using a conventional scheme based on an ADC. According to the energy consumption detailed in [21] with technologies available in 2008, the consumption would be around $10 \mathrm{~nJ}$, which is almost three orders of magnitude lower. Thus, the energy consumption is a serious design issue when the $\mu \mathrm{C}$ does not provide a proper ADC and we have to use a direct interface circuit instead. In this case, the optimization of energy consumption might play an important role in the design process.

\section{REFERENCES}

[1] A. P. Sample, D. J. Yeager, P. S. Powledge, A. V. Mamishev, and J. R. Smith, "Design of an RFID-based battery-free programmable sensing platform," IEEE Trans. Instrum. Meas., vol. 57, no. 11, pp. 2608-2615, Nov. 2008.

[2] J. Pelegrí-Sebastiá, E. García-Breijo, J. Ibañez, T. Sogorb, N. Laguarda-Miro, and J. Garrigues, "Low-cost capacitive humidity sensor for application within flexible RFID labels based on microcontroller systems," IEEE Trans. Instrum. Meas., vol. 61, no. 2, pp. 545-553, Feb. 2012.

[3] E. Sardini and M. Serpelloni, "Self-powered wireless sensor for air temperature and velocity measurements with energy harvesting capability," IEEE Trans. Instrum. Meas., vol. 60, no. 5, pp. 1838-1844, May 2011.

[4] R. Casanella and R. Pallàs-Areny, "On the design of low-power signal conditioners for resistive sensors," in Proc. 19th IMEKO World Congr. Fundam. Appl. Metrol., 2009, pp. 787-791.

[5] Z. Kokolanski, C. Gavrovski, V. Dimcev, and M. Makraduli, "Simple interface for resistive sensors based on pulse width modulation," IEEE Trans. Instrum. Meas., vol. 62, no. 11, pp. 2983-2992, Nov. 2013.

[6] D. Cox, "Implementing ohmmeter/temperature sensor," Microchip Technol., Inc., Chandler, AZ, USA, Appl. Note AN512, 1994.

[7] L. Bierl, "MSP430 family mixed-signal microcontroller application reports," Texas Instrum., Dallas, TX, USA, Tech. Rep. SLAA024, 1996.

[8] L. Bengtsson, "Direct analog-to-microcontroller interfacing," Sens. Actuators A, Phys., vol. 179, pp. 105-113, Jun. 2012.

[9] F. Reverter, "The art of directly interfacing sensors to microcontrollers," J. Low Power Electron. Appl., vol. 2, no. 4, pp. 265-281, Nov. 2012.

[10] F. Reverter and Ò. Casas, "Interfacing differential resistive sensors to microcontrollers: A direct approach," IEEE Trans. Instrum. Meas., vol. 58, no. 10, pp. 3405-3410, Oct. 2009.

[11] E. Sifuentes, O. Casas, F. Reverter, and R. Pallàs-Areny, "Direct interface circuit to linearise resistive sensor bridges," Sens. Actuators A, Phys. vol. 147, no. 1, pp. 210-215, 2008.

[12] F. Reverter, "Power consumption in direct interface circuits," IEEE Trans. Instrum. Meas., vol. 62, no. 2, pp. 503-509, Feb. 2013.

[13] F. M. L. van der Goes, "Low-cost smart sensor interfacing," Ph.D. dissertation, Dept. Elect. Eng., Delft Univ. Technol., Delft, The Netherlands, 1996.

[14] F. Reverter and R. Pallàs-Areny, Direct Sensor-to-Microcontroller Interface Circuits: Design and Characterisation. Barcelona, Spain: Marcombo, 2005.

[15] Z. Kokolanski, C. Gavrovski, V. Dimcev, and M. Makradul, "Hardware techniques for improving the calibration performance of direct resistive sensor-to-microcontroller interface," Metrol. Meas. Syst., vol. 20, no. 4 pp. 529-542, 2013.

[16] C. F. Dietrich, Uncertainty, Calibration and Probability: The Statistics of Scientific and Industrial Measurement, 2nd ed. New York, NY, USA: Adam Hilger, 1991.

[17] L. Benini, A. Bogliolo, and G. De Micheli, "A survey of design techniques for system-level dynamic power management," IEEE Trans. Very Large Scale Integr. (VLSI) Syst., vol. 8, no. 3, pp. 299-316, Jun. 2000

[18] C. Cockrill, "Understanding Schmitt triggers," Texas Instrum., Dallas, TX, USA, Appl. Rep. SCEA046, Sep. 2011. 
[19] "MSP430X1XX family user's guide," Texas Instrum., Dallas, TX, USA, Rep. SLAU049F, 2006.

[20] J. Jordana, F. Reverter, and R. Pallàs-Areny, "Uncertainty in resistance measurements based on microcontrollers with embedded time counters," in Proc. 20th IEEE Instrum. Meas. Technol. Conf. (IMTC), Vail, CO, USA, May 2003, pp. 1078-1082.

[21] B. Murmann, "A/D converter trends: Power dissipation, scaling and digitally assisted architectures," in Proc. IEEE Custom Intergr. Circuits Conf. (CICC), Sep. 2008, pp. 105-112.

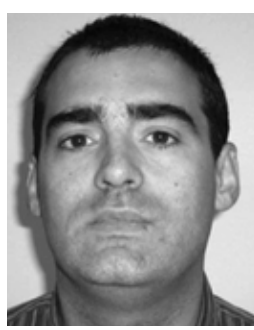

Oscar López-Lapeña received the M.S. degrees in physics and electronics engineering from the Universitat de Barcelona, Barcelona, Spain, in 1994 and 1996, respectively, and the Ph.D. degree in electronics engineering from the Universitat Politècnica de Catalunya (UPC), Barcelona, in 2000.

He has been an Associate Professor with UPC since 2002. He has authored several journal and conference papers and holds two patents in energy harvesting. His current research interests include low-power converters, control theory, and energy harvesting.

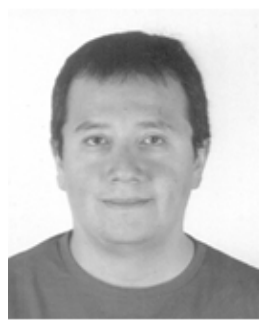

Ernesto Serrano-Finetti was born in Lima, Peru, in 1972. He received the Ingeniero en Electrónica degree from the Pontificia Universidad Católica del Perú, Lima, in 1998, and the Ph.D. degree in biomedical engineering from the Universitat Politècnica de Catalunya (UPC), Barcelona, Spain, in 2003.

$\mathrm{He}$ has been an Assistant Professor with UPC since 2002, where he teaches courses on electronic systems and instrumentation related areas. His current research interests include low-power analog design for autonomous sensors, sensor interfaces, sensors based on electrical impedance measurements, noninvasive physiological sensors and measurements, and environmental measurements.

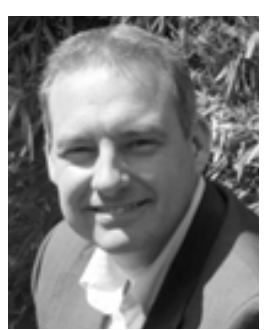

Oscar Casas (''93-A'99-M'05) received the Ingeniero de Telecomunicación and Doctor Ingeniero de Telecomunicación degrees from the Universitat Politècnica de Catalunya (UPC), Barcelona, Spain, in 1994 and 1998, respectively.

$\mathrm{He}$ is currently an Associate Professor of Electronic Engineering with UPC and teaches courses in several areas of electronic instrumentation. His current research interests include sensor interfaces, autonomous sensors, electronic instrumentation, and noninvasive physiological measurements and sensors based on electrical impedance measurements. 